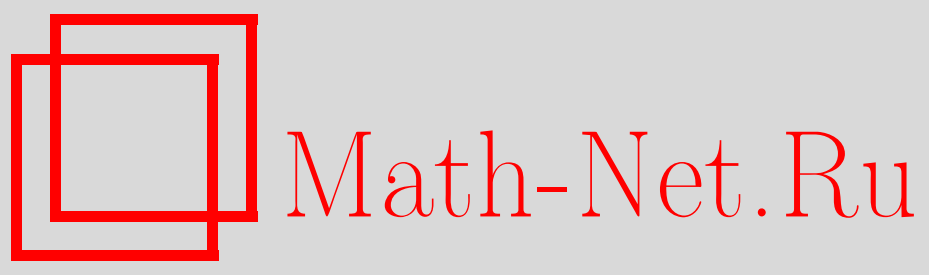

М. А. Соловьев, Об аксиоматических формулировках нелокальной и некоммутативной теорий поля, ТMФ, 2006, том 147, номер 2, 257-269

DOI: https://doi.org/10.4213/tmf1962

Использование Общероссийского математического портала Math-Net.Ru подразумевает, что вы прочитали и согласны с пользовательским соглашением http://www . mathnet.ru/rus/agreement

Параметры загрузки:

IP: 34.229 .108 .108

26 апреля 2023 г., 14:08:43

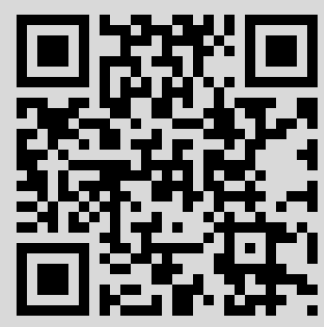




\title{
ОБ АКСИОМАТИЧЕСКИХ ФОРМУЛИРОВКАХ НЕЛОКАЛЬНОЙ И НЕКОММУТАТИВНОЙ ТЕОРИЙ ПОЛЯ
}

\begin{abstract}
Анализируются функционально-аналитические аспекты аксиоматических формулировок нелокальной и некоммутативной теорий поля. Полностью выяснено соотношение между условием асимптотической коммутативности, обеспечивающим СРТ-симметрию и нормальную связь спина со статистикой для нелокальных полей, и свойствами регулярности запаздывающих функций Грина в импульсном пространстве, необходимыми для построения матрицы рассеяния и вывода редукционных формул. В основе этого результата лежит обобщение теоремы Пэли-Винера-Шварца на аналитические функционалы. Обсуждается возможность использования аналитических пробных функций при распространении аксиом Вайтмана на некоммутативную теорию поля, в которой причинная структура со световым конусом заменяется структурой со световым клином. Объясняются существенные особенности вывода теорем о СРТ-симметрии и связи спина со статистикой в такой расширенной формулировке.
\end{abstract}

Ключевые слова: нелокальные квантовые поля, причинность, некоммутативная теория поля, вайтмановские функции, аналитические функционалы, теорема Пэли-ВинераШварца.

\section{1. ВВЕДЕНИЕ}

В данной статье рассматриваются вопросы, возникающие при распространении аксиом Вайтмана на нелокальные и некоммутативные квантовые теории поля (КТП) и связанные с привлечением для этой цели новых классов обобщенных функций, более широких, чем распределения умеренного роста, принятые в обычном формализме [1]-[3]. Пожалуй, наиболее часто предлагалось использовать функционалы, определенные на пространстве $S^{0}$ аналитических пробных функций. Это пространство есть не что иное, как фурье-образ пространства Шварца $\mathcal{D}$ гладких функций с компактным носителем, и оно может служить функциональной областью определения для полей с произвольным высокоэнергетическим поведением. Первый из обсуждаемых ниже вопросов имеет давнюю историю. В работе [4] Штейнман предложил заменить аксиому локальной коммутативности, которая утрачивает смысл

* Физический институт им. П. Н. Лебедева РАН, Москва, Россия. E-mail: soloviev@lpi.ru

4 Теоретическая и математическая физика, т. 147, № 2, 2006 г. 
для полей, заданных на $S^{0}$, условиями регулярности запаздывающих функций Грина в импульсном пространстве, обеспечивающими существование $S$-матрицы и достаточными для построения теории рассеяния. Впоследствии было найдено обобщение условия микропричинности в координатном представлении. Это обобщение, получившее название "асимптотическая коммутативность", обеспечивает нормальную связь спина со статистикой и существование оператора СРТ-симметрии в нелокальной теории, см. работу [5] и ссылки в ней. Асимптотическая коммутативность формулируется как требование непрерывности коммутаторов наблюдаемых полей в топологии некоторого пространства, родственного $S^{0}$ и ассоциированного с замкнутым световым конусом. В [6] для обобщенных функций, определенных на $S^{0}$, доказана теорема типа теоремы Пэли-Винера-Шварца, которая позволяет полностью выяснить соотношение между асимптотической коммутативностью и условиями Штейнмана. Однако для этого, как мы увидим, потребуется распространение этой теоремы на полилинейные функционалы.

Эта же математическая техника оказывается полезной при анализе предложенных недавно аксиоматических формулировок некоммутативной теории поля. Как отмечено в [7], такая аксиоматизация отчасти преждевременна, поскольку нет еще достаточного числа хорошо изученных инструктивных примеров, как нет и скольконибудь единой точки зрения на ситуацию с пуанкаре-симметрией, причинностью и унитарностью в некоммутативной теории поля. Зачастую под этим названием подразумеваются совершенно разные теории с разным физическим содержанием. Мы прокомментируем здесь попытки распространения аксиом Вайтмана на те из них, которые возникают из теории струн в пределе Зайберга-Виттена [8], и характерной чертой которых является замена причинной структуры со световым конусом на структуру со световым клином. Такая модификация условия микропричинности предлагалась, например, в [9] и подробно обсуждалась в [10], [11]. Анализ этой формулировки интересен и с методической точки зрения, поскольку показывает, что для вывода теорем о связи спина со статистикой и теорем о СРТ-симметрии нет необходимости использовать теорему Баргмана-Холла-Вайтмана в полном объеме, а достаточно применить ее простейший вариант для двумерного пространства-времени, для которого комплексная группа Лоренца совпадает с группой $\mathbb{C}^{*}=\mathbb{C} \backslash\{0\}$ комплексных чисел. В [9], [11] подчеркивалось, что распределения умеренного роста вряд ли достаточны для развиваемой авторами теории из-за сингулярностей, связанных с ультрафиолетово-инфракрасным смешиванием, и ввиду экспоненциального роста корреляционных функций в некоммутативных направлениях импульсного пространства. В [12] предложено использовать вместо распределений умеренного роста обобщенные функции класса $S^{\prime 0}$. Во второй части статьи мы объясним некоторые особенности доказательства теорем о СРТ-симметрии и связи спина со статистикой в рамках такого формализма и покажем, что наиболее простой путь их вывода состоит в обобщении теоремы единственности, полученной в [13] для распределений с носителем в остром конусе, на случай носителя в остром клине.

В следующем разделе излагаются необходимые предварительные сведения об используемых функциональных пространствах и вводятся ключевые для дальнейше- 
го понятия несущих конусов обобщенной функции класса $S^{\prime 0}\left(\mathbb{R}^{d}\right)$ и полилинейного функционала, заданного на $S^{0}\left(\mathbb{R}^{d}\right) \times \cdots \times S^{0}\left(\mathbb{R}^{d}\right)$. В разделе 3 сформулированы условия регулярности Штейнмана и кратко охарактеризованы основные свойства запаздывающих произведений нелокальных квантовых полей. В разделе 4 для полилинейных функционалов доказана теорема типа теоремы Пэли-Винера-Шварца и с ее помощью установлена связь условий Штейнмана с условием асимптотической коммутативности. В разделе 5 получено обобщение теоремы единственности, доказанной в работе [13], на распределения с носителем в остром клине. Оно позволяет вывести в теории с двумя некоммутирующими пространственными координатами аналоги теорем о связи спина со статистикой и СРТ-симметрии по той же схеме (см. работу [5]), что и в нелокальной КТП. Раздел 6 содержит заключительные замечания.

\section{2. ПРЕДВАРИТЕЛЬНЫЕ СВЕДЕНИЯ}

Напомним, что пространство $S^{0}$ состоит из целых аналитических функций, удовлетворяющих неравенствам

$$
|f(x+i y)| \leqslant C_{N}(1+|x|)^{-N} e^{B|y|}, \quad N=0,1,2, \ldots,
$$

где константы $C_{N}$ и $B$ зависят от $f[14]$. Для построения теории поля с такими пробными функциями оказывается необходимым использовать родственные $S^{0}$ пространства, ассоциированные с конусами в $\mathbb{R}^{d}$. Если $U \subset \mathbb{R}^{d}$ - открытый конус, то пространство $S^{0}(U)$ определяется как объединение счетно-нормированных пространств $S^{0, b}(U), \quad b>0$, состоящих из целых функций с конечными нормами вида

$$
\begin{gathered}
\|f\|_{U, B, N}=\sup _{z \in \mathbb{C}^{d}}|f(z)| \prod_{j=1}^{d}\left(1+\left|x_{j}\right|\right)^{N} e^{-B d(x, U)-B|y|}, \\
B>b, \quad N=0,1,2, \ldots,
\end{gathered}
$$

где $z=x+i y$ и $d(x, U)=\inf _{\xi \in U}|x-\xi|-$ расстояние от точки $x$ до $U$. Ясно, что $S^{0}(U)$ непрерывно вложено в $S^{0}\left(U^{\prime}\right)$ при $U \supset U^{\prime}$. В качестве нормы в $\mathbb{R}^{d}$ мы возьмем $|x|=\sum_{j}\left|x_{j}\right|$. Тогда норма (1) обладает свойством мультипликативности, важным при рассмотрении тензорных произведений и используемым ниже: если $f_{1} \in S^{0}\left(U_{1}\right)$ и $f_{2} \in S^{0}\left(U_{2}\right)$, то справедлива формула

$$
\left\|f_{1} \otimes f_{2}\right\|_{U_{1} \times U_{2}, B, N}=\left\|f_{1}\right\|_{U_{1}, B, N} \cdot\left\|f_{2}\right\|_{U_{2}, B, N} .
$$

Пространство линейных непрерывных функционалов на $S^{0}$ мы обозначаем через $S^{\prime 0}$ и говорим, что замкнутый конус $K$ является несущим для $v \in S^{\prime 0}$, если функционал $v$ допускает непрерывное продолжение на каждое пространство $S^{0}(U)$, где $U \supset K \backslash\{0\}$ (это включение обозначается также как $U \ni K$ ). Это свойство равносильно тому, что $v$ имеет непрерывное продолжение на пространство индуктивного предела

$$
S^{0}(K)=\underset{U \ni K}{\lim _{U \ni K}} S^{0}(U) .
$$


Пусть $w$ - раздельно непрерывная (непрерывная по каждому аргументу в отдельности) полилинейная форма на $\underbrace{S^{0}\left(\mathbb{R}^{d}\right) \times \cdots \times S^{0}\left(\mathbb{R}^{d}\right)}_{n}$ и пусть $K=K_{1} \times \cdots \times K_{n}$, где $K_{j}$ - замкнутые конусы в $\mathbb{R}^{d}$. Естественно назвать $K$ несущим конусом формы $w$, если каждый конус $K_{j}$ является несущим для всех линейных функционалов на $S^{0}\left(\mathbb{R}^{d}\right)$, определяемых $w\left(f_{1}, \ldots, f_{n}\right)$ при фиксированных $f_{i} \in S^{0}\left(\mathbb{R}^{d}\right), i \neq j$. В силу теоремы Шварца о ядре для каждой формы $w$ существует единственный линейный функционал $v \in S^{\prime 0}\left(\mathbb{R}^{d n}\right)$ такой, что

$$
w\left(f_{1}, \ldots, f_{n}\right)=v\left(f_{1} \otimes \cdots \otimes f_{n}\right) .
$$

В теоретико-полевом контексте это означает возможность отождествления вакуумных средних полей с некоторыми обобщенными функциями, как и в обычном формализме [1]-[3]. Для анализа указанных во введении вопросов будут использованы следующие факты.

Теорема 1. Пространство $S^{0}\left(\mathbb{R}^{d}\right)$ плотно в любом пространстве $S^{0}(U)$, где $U$ - открытый конус в $\mathbb{R}^{d}$.

ТеОРема 2. У каждого элемента $S^{\prime 0}\left(\mathbb{R}^{d}\right)$ имеется наименьший замкнутый несущий конус.

Теорема 3. Если $v \in S^{\prime 0}\left(\mathbb{R}^{d}\right)$ - функционал с острым несущим конусом $K$, то он имеет преобразование Лапласа $\mathbf{u}(\zeta)=\left(v, e^{i z \zeta}\right)$, являющееся аналитической функцией в трубчатой области $T^{V}=\mathbb{R}^{d}+i V$, где $V$ - внутренность дуального конуса $K^{*}=\{\eta: x \eta \geqslant 0 \quad \forall x \in K\}$, и удовлетворяющее условию

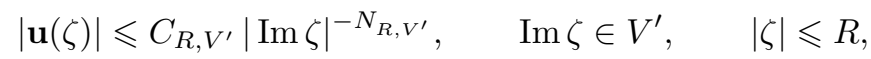

при любом $R>0$ и любом конусе $V^{\prime} \Subset V$. Если $\operatorname{Im} \zeta \rightarrow 0$ внутри фиксированного $V^{\prime}$, то $\mathbf{u}(\zeta)$ стремится $\kappa$ фуръе-образу $v$ в топологии $\mathcal{D}^{\prime}$. Обратно, любая функция, аналитическая в $T^{V}$, где $V$ - открытый конус в $\mathbb{R}^{d}$, и удовлетворяющая условию (4), есть преобразование Фуръе-Лапласа некоторого элемента $S^{\prime 0}\left(V^{*}\right)$.

ТеОрема 4. Конус $K_{1} \times \cdots \times K_{n}$ является несущим для раздельно непрерывной полилинейной формы на $\underbrace{S^{0}\left(\mathbb{R}^{d}\right) \times \cdots \times S^{0}\left(\mathbb{R}^{d}\right)}_{n}$ в том и только том случае, когда порождаемая ею обобщенная функиия на $\mathbb{R}^{d n}$ имеет непрерывное продолжение на пространство

$$
S^{0}\left(K_{1}, \ldots, K_{n}\right)=\underset{U_{1} \ni K_{1}, \ldots, U_{n} \ni K_{n}}{\lim _{\longrightarrow}} S^{0}\left(U_{1} \times \cdots \times U_{n}\right) .
$$

Эти теоремы аналогичны установленным ранее для другого функционального класса $S_{\alpha}^{\prime 0}$, но их вывод несколько более труден из-за того, что топологическая структура $S^{0}(U)$ сложнее, чем у $S_{\alpha}^{0}(U)$. Теорема 1 получена в [13] путем сведения к теореме плотности для $S_{\alpha}^{0}(U)$. Теоремы 2 и 3 доказаны в [6]. Определение $(5)$ является аналогом определения, предложенного в [15] для $S_{\alpha}^{0}\left(K_{1}, \ldots, K_{n}\right)$. Теорема 4 
доказывается также, как и теорема 3 в [16], но с использованием теоремы 1 из работы [17] вместо леммы 5 в [16]. Для рассматриваемых приложений существенно, что пространство $S^{0}\left(K_{1}, \ldots, K_{n}\right)$ не совпадает с $S^{0}\left(K_{1} \times \cdots \times K_{n}\right)$. Последнее есть подпространство $S^{0}\left(K_{1}, \ldots, K_{n}\right)$, но оно является собственным, за исключением двух тривиальных случаев $K_{j}=\{0\}$ и $K_{j}=\mathbb{R}^{d}$ при всех $j$, см. [15], [16]. Если функционал $v \in S^{\prime 0}\left(K_{1} \times \cdots \times K_{n}\right)$ допускает непрерывное продолжение на $S^{0}\left(K_{1}, \ldots, K_{n}\right)$, мы говорим, что составной конус $K_{1} \times \cdots \times K_{n}$ является для $v$ строго несущим.

\section{3. УСЛОВИЕ ШТЕЙНМАНА}

Рассмотрим простейший случай скалярного нейтрального нелокального поля $\phi$, считая его операторной обобщенной функцией, определенной на $S^{0}\left(\mathbb{R}^{4}\right)$ вместо пространства $S\left(\mathbb{R}^{4}\right)$ гладких быстроубывающих функций, обычно используемого в локальной КТП [1]-[3]. Предположим, что ф удовлетворяет всем аксиомам Вайтмана, за исключением условия микропричинности, формулировка которого при таких пробных функциях невозможна. Общую плотную область определения операторов $\phi(f), \quad f \in S^{0}\left(\mathbb{R}^{4}\right)$, в гильбертовом пространстве состояний обозначим через $D$, а унитарное представление в нем группы Пуанкаре - через $U(\Lambda, a)$. Как и в [4], предположим существование запаздывающих произведений полевых операторов $R\left(x ; x_{1}, \ldots, x_{n}\right), \quad n=1,2, \ldots$, обладающих следующими характеристическими свойствами:

1) операторы

$$
R(f)=\int R\left(x ; x_{1}, \ldots, x_{n}\right) f\left(x, x_{1}, \ldots, x_{n}\right) d x d x_{1} \ldots d x_{n}, \quad f \in S^{0}\left(\mathbb{R}^{4(n+1)}\right),
$$

определены на области $D$ и отображают ее в себя;

2) операторы $R(f)$ эрмитовы при вещественных $f$;

3) $R(x)=\phi(x)$;

4) $R$-произведение симметрично по переменным $x_{1}, \ldots, x_{n}$;

5) имеет место равенство

$$
\begin{aligned}
& R\left(x ; y, x_{1}, \ldots, x_{n}\right)-R\left(y ; x, x_{1}, \ldots, x_{n}\right)= \\
& \quad=-i \sum\left[R\left(x ; x_{i_{1}}, \ldots, x_{i_{\alpha}}\right), R\left(y ; x_{i_{\alpha+1}}, \ldots, x_{i_{n}}\right)\right],
\end{aligned}
$$

где суммирование ведется по всем разбиениям $\left\{x_{1}, \ldots, x_{n}\right\}$ на два множества;

6) $R\left(\Lambda x+a ; \Lambda x_{1}+a, \ldots, \Lambda x_{n}+a\right)=U(\Lambda, a) R\left(x ; x_{1}, \ldots, x_{n}\right) U(\Lambda, a)^{-1}$.

В локальной теории поля определяющим является условие причинности

7) $\operatorname{supp} R\left(x ; x_{1}, \ldots, x_{n}\right) \subset \mathbb{K}_{n}$, где

$$
\begin{aligned}
\mathbb{K}_{n} & =\mathbb{R}^{4} \times \overline{\mathbb{V}}_{-} \times \cdots \times \overline{\mathbb{V}}_{-}=\left\{\left(x, x_{1}, \ldots, x_{n}\right):\left(x_{j}-x\right) \in \overline{\mathbb{V}}_{-}, \quad j=1, \ldots, n\right\} \\
\text { и } \overline{\mathbb{V}}_{-} & =\left\{\xi: \xi^{2} \geqslant 0, \xi^{0} \leqslant 0\right\} \text { - замкнутый нижний конус. }
\end{aligned}
$$

Штейнман предложил заменить свойство 7 условием регулярности запаздывающих произведений в $p$-пространстве. Преобразование Фурье матричного элемента $\left\langle\Phi, R\left(x ; x_{1}, \ldots, x_{n}\right) \Psi\right\rangle$, где $\Phi, \Psi \in D$, можно рассматривать как распределение по переменным $p_{1}, \ldots, p_{n}$ и $P=p+p_{1}+\cdots+p_{n}$. Если обозначить через $\chi\left(p_{1}, \ldots, p_{n}\right)$ 
распределение, получающееся в результате его интегрирования с пробной функцией, зависящей лишь от $P$, то это условие регулярности записывается следующим образом ${ }^{1)}$.

УСловиЕ $\mathcal{R}$. Функция $\chi\left(p_{1}, \ldots, p_{n}\right)$ аналитична в трубчатой области

$$
\mathbb{T}_{-}^{n}=\left\{\left(p_{1}, \ldots, p_{n}\right): \operatorname{Im} p_{j} \in \mathbb{V}_{-}, \quad j=1, \ldots, n\right\}
$$

Для всякого компакта $Q \subset \mathbb{R}^{4 n}$ и для любых $\gamma>1$ u $R>0$ найдутся положительная константа $C$ и неотрицательные целые числа $N_{j}$ такие, что

$$
\left|\chi\left(p_{1}, \ldots, p_{n}\right)\right| \leqslant C \prod_{j=1}^{n}\left|\operatorname{Im} p_{j 0}\right|^{-N_{j}}
$$

$n p u\left(p_{1}, \ldots, p_{n}\right) \in \mathbb{T}_{-}^{n}, \quad\left(\operatorname{Re} p_{1}, \ldots, \operatorname{Re} p_{n}\right) \in Q u \gamma\left|\operatorname{Im} \mathbf{p}_{j}\right| \leqslant\left|\operatorname{Im} p_{j 0}\right| \leqslant R, j=1, \ldots, n$ (здесъ $p_{j 0}$-0-компонента 4-вектора $p_{j}, \mathbf{p}_{j}$ - его пространственная часть).

Согласно этому условию свойства аналитичности запаздывающих произведений нелокальных полей в $p$-пространстве являются такими же, как и у запаздывающих произведений в обычном локальном формализме [3]. Ограничение (8) обеспечивает существование граничного значения аналитической функции $\chi$ как элемента пространства распределений Шварца $\mathcal{D}^{\prime}$. Однако, в отличие от локальной теории, при этом не накладывается никаких ограничений на поведение $\chi$ при больших $p_{j}$, вещественных или мнимых.

В работе [4] не ставилась задача выяснения смысла условия $\mathcal{R}$ в координатном представлении. Автор этой работы подчеркивал, что считает его слишком сложным и далеким от прямой физической интерпретации, чтобы принимать в качестве основного постулата, и предлагал рассматривать его просто как технически удобное свойство, достаточное для построения для нелокальных полей последовательного формализма теории рассеяния, включающего доказательство существования асимптотических состояний и $S$-матрицы. Основной результат работы [4] состоит в том, что при условии $\mathcal{R}$ редукционные формулы Лемана-СиманзикаЦиммермана для элементов $S$-матрицы соблюдаются в точности в том же виде, что и в локальной КТП. Мы сейчас покажем, что в $x$-пространстве это условие является естественным обобщением свойства 7) носителей запаздывающих произведений локальных полей и, тем самым, подходящим кандидатом на условие причинности для нелокальной теории поля. Отметим еще, что из $R$-произведений с помощью обычных рекурсивных соотношений [3] можно построить аналог хронологического $T$-произведения и что из условия $\mathcal{R}$ вытекает существование единой аналитической функции, граничными значениями которой в разных областях являются преобразования Фурье запаздывающей и причинной функций Грина $\left\langle\Psi_{0}, R\left(x ; x_{1}, \ldots, x_{n}\right) \Psi_{0}\right\rangle$ и $\left\langle\Psi_{0}, T\left(x ; x_{1}, \ldots, x_{n}\right) \Psi_{0}\right\rangle$, где $\Psi_{0}$ - вакуумное состояние.

\footnotetext{
1) В [4] дана формулировка для обобщенных запаздывающих произведений, но мы для простоты ограничиваемся частным случаем обычных запаздывающих произведений.
} 


\section{4. СВЯЗЬ С АСИМПТОТИЧЕСКОЙ КОММУТАТИВНОСТЬЮ}

Теорема 4 показывает, что пространство раздельно непрерывных полилинейных форм на $S^{0}\left(\mathbb{R}^{d}\right) \times \cdots \times S^{0}\left(\mathbb{R}^{d}\right)$ с составным несущим конусом $K_{1} \times \cdots \times K_{n}$ может быть отождествлено с пространством, сопряженным к пространству (5). Пусть $V_{1}, \ldots, V_{n}$ - открытые конусы в $\mathbb{R}^{d}$. Обозначим через $\mathcal{A}_{0}\left(V_{1}, \ldots, V_{n}\right)$ пространство функций, аналитических в трубчатой области $T^{V}=\mathbb{R}^{d n}+i V$, где $V=V_{1} \times \cdots \times V_{n}$, и удовлетворяющих условию

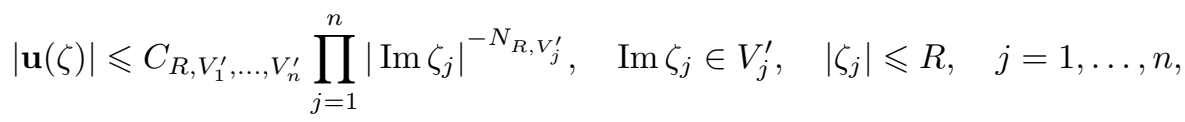

при любом $R>0$ и любых конусах $V_{j}^{\prime} \Subset V_{j}$. Очевидно, $\mathcal{A}_{0}\left(V_{1}, \ldots, V_{n}\right)$ является алгеброй по умножению.

Теорема 5. Преобразование Лапласа $\mathcal{L}: v \rightarrow\left(v, e^{i z \zeta}\right)$ устанавливает изоморбизм между пространством $S^{\prime 0}\left(V_{1}^{*}, \ldots, V_{n}^{*}\right)$ u алгеброй $\mathcal{A}_{0}\left(V_{1}, \ldots, V_{n}\right) . \quad$ Если $\operatorname{Im} \zeta \rightarrow 0$ внутри фиксированного конуса $V_{1}^{\prime} \times \cdots \times V_{n}^{\prime}$, где $V_{j}^{\prime} \Subset V_{j}$, то функиия $(\mathcal{L} v)(\zeta)$ стремится $\kappa$ фуръе-образу $v$ в топологии $\mathcal{D}^{\prime}\left(\mathbb{R}^{d n}\right)$.

ДокАЗАтЕЛЬСтво. Поскольку $S^{\prime 0}\left(V_{1}^{*}, \ldots, V_{n}^{*}\right) \subset S^{\prime 0}\left(V^{*}\right)$, мы можем воспользоваться теоремой 3 , формулировка которой получается из формулировки теоремы 5 при $n=1$. Теорема 3 показывает, в частности, что любой функционал из $S^{\prime 0}\left(V^{*}\right)$ имеет преобразование Лапласа, аналитическое в области $T^{V}$. Ограничение (9) сильнее, чем ограничение (4), которое справедливо для произвольного элемента $S^{\prime 0}\left(V^{*}\right)$, но выводится по той же схеме на основании оценки

$$
|\mathcal{L} v(\zeta)|=\left|\left(v, e^{i z \zeta}\right)\right| \leqslant\|v\|_{U, B, N}\left\|e^{i z \zeta}\right\|_{U, B, N} .
$$

Здесь $U=U_{1} \times \cdots \times U_{n}, U_{j}$ - любые открытые конусы такие, что $V_{j}^{*} \Subset U_{j}, B$ сколь угодно велико, и $N$ зависит, вообе говоря, от $B$ и $U$. Благодаря свойству (2) мы имеем равенство

$$
\left\|e^{i z \zeta}\right\|_{U, B, N}=\prod_{j}\left\|e^{i z_{j} \zeta_{j}}\right\|_{U_{j}, B, N}
$$

где каждый сомножитель оценивается точно так же, как и норма экспоненты в [6], что и приводит к (9).

Пусть теперь $u$ - граничное значение функции $\mathbf{u}$ со свойством (9), существующее в $\mathcal{D}^{\prime}\left(\mathbb{R}^{d n}\right)$ согласно теореме 3.1 .15 в [18]. Теорема 3 показывает, что конус $V_{1}^{*} \times \cdots \times V_{n}^{*}$ является несущим для полилинейной формы на $S^{0}\left(\mathbb{R}^{d}\right) \times \cdots \times S^{0}\left(\mathbb{R}^{d}\right)$, определяемой обратным преобразованием Фурье распределения $u$. Применение теоремы 4 завершает доказательство.

Переформулировав условие регулярности функций Грина нелокальной теории поля в терминах теоремы 5, мы получаем следующий результат.

Теорема 6. Условие $\mathcal{R}$ равносильно тому, что в $x$-пространстве конус (6) является строго несущим для функиионалов на $S^{0}\left(\mathbb{R}^{4(n+1)}\right)$, задаваемых матричными элементами $\left\langle\Phi, R\left(x ; x_{1}, \ldots, x_{n}\right) \Psi\right\rangle, \Phi, \Psi \in D$. 
Подробнее, условие $\mathcal{R}$ означает, что все эти функционалы допускают непрерывное продолжение на любое пространство $S^{0}\left(U_{\gamma}\right)$, где

$$
U_{\gamma}=\left\{\left(x, x_{1}, \ldots, x_{n}\right):\left|\mathbf{x}-\mathbf{x}_{j}\right|<\gamma\left(x^{0}-x_{j}^{0}\right), \quad j=1, \ldots, n\right\}, \quad \gamma>1 .
$$

В частности, совместно со свойством 5 из раздела 3 условие $\mathcal{R}$ приводит к тому, что конус $\mathbb{R}^{4} \times \overline{\mathbb{V}}=\left\{(x, y) \in \mathbb{R}^{8}:(x-y)^{2} \geqslant 0\right\}$ является строго несущим для матричных элементов коммутатора

$$
\langle\Phi,[\phi(x), \phi(y)] \Psi\rangle=\langle\Phi,(R(x, y)-R(y, x)) \Psi\rangle, \quad \Phi, \Psi \in D .
$$

Таким образом, теория удовлетворяет условию асимптотической коммутативности (см. работу [5]), обеспечивающему СРТ-инвариантность и нормальную связь спина со статистикой.

\section{5. ОБ АКСИОМАТИЧЕСКОЙ ФОРМУЛИРОВКЕ НЕКОММУТАТИВНОЙ КТП}

Мы рассмотрим теорию с некоммутативностью между двумя пространственными координатами, а именно, с коммутационным соотношением

$$
\left[\hat{x}^{\mu}, \hat{x}^{\nu}\right]=i \theta^{\mu \nu}
$$

где $\theta^{23}=-\theta^{32}$ - постоянный параметр некоммутативности, а все остальные элементы матрицы $\theta^{\mu \nu}$ равны нулю. Ясно, что наибольшей подгруппой группы Лоренца, оставляющей соотношение $(10)$ неизменным, является $O(1,1) \times S O(2)$, связная компонента которой есть произведение группы $S O_{0}(1,1)$ гиперболических поворотов в плоскости $\left(x^{0}, x^{1}\right)$ и группы $S O(2)$ вращений в плоскости $\left(x^{2}, x^{3}\right)$. Основная идея работы [9] заключается в согласовании аксиоматики Вайтмана с этой остаточной симметрией. Приняв такую точку зрения, мы считаем, что в гильбертовом пространстве состояний реализуется унитарное представление $U(\Lambda, a)$ связной группы

$$
\left[S O_{0}(1,1) \times S O(2)\right] \rtimes \mathcal{T}_{4},
$$

где $\mathcal{T}_{4}$ - подгруппа трансляций, и предполагаем существование и единственность вакуумного состояния $\Psi_{0}$, инвариантного относительно $U(I, a)=e^{i a^{\mu} P_{\mu}}$. Предложенная в [9] модификация спектрального условия состоит в требовании принадлежности спектра оператора энергии-импульса $P$ верхнему световому клину:

$$
\operatorname{Spec} P \subset \overline{\mathbb{V}}_{\mathrm{c}+} \times \mathbb{R}^{2}=\left\{p \in \mathbb{R}^{4}: p_{\mathrm{c}}^{2}=p_{0}^{2}-p_{1}^{2} \geqslant 0, \quad p_{0} \geqslant 0\right\} .
$$

Здесь и далее мы обозначаем индексом с величины, относящиеся к двумерному коммутативному подпространству. Аналогичным образом постулат микропричинности заменяется условием исчезновения коммутаторов полей (или антикоммутаторов в случае ненаблюдаемых полей) $\left[\phi_{\iota}(x), \phi_{\iota^{\prime}}\left(x^{\prime}\right)\right]_{\mp}$ вне замкнутого клина

$$
\overline{\mathbb{V}}_{\mathrm{c}} \times \mathbb{R}^{6}=\left\{\left(x, x^{\prime}\right):\left(x-x^{\prime}\right)_{\mathrm{c}}^{2} \geqslant 0\right\} .
$$


Мы ограничимся рассмотрением комплексного скалярного поля $\phi$ с законом преобразования

$$
U(\Lambda, a) \phi(f) U^{-1}(\Lambda, a)=\phi\left(f_{(\Lambda, a)}\right),
$$

где $f_{(\Lambda, a)}(x)=f\left(\Lambda^{-1}(x-a)\right)$, и прокомментируем обсуждавшуюся в [12] возможность обобщения этой аксиоматической схемы путем использования аналитических пробных функций из пространства $S^{0}$.

Сначала отметим следствие условий (12), (14), справедливое вне зависимости от формулировки причинности. Ввиду трансляционной инвариантности $n$-точечное вакуумное среднее

$$
\mathcal{W}\left(x_{1}, \ldots, x_{n}\right)=\left\langle\Psi_{0}, \phi^{(*)}\left(x_{1}\right) \ldots \phi^{(*)}\left(x_{n}\right) \Psi_{0}\right\rangle
$$

где $\phi^{(*)}$ есть либо поле $\phi$, либо эрмитово сопряженное поле $\phi^{*}$, может быть отождествлено с обобщенной функцией $W \in S^{\prime 0}\left(\mathbb{R}^{4(n-1)}\right)$ от разностных переменных $\xi_{j}=x_{j}-x_{j+1}, \quad j=1, \ldots, n-1$. Лемма 4 в [5] показывает, что конус $K \subset \mathbb{R}^{4(n-1)}$ является несущим для функции Вайтмана $W$ в том и только том случае, когда его прообраз $K \times \mathbb{R}^{4}$ в $\mathbb{R}^{4 n}$ является строго несущим для $\mathcal{W}$, а принадлежность $W \in S^{\prime 0}\left(K_{1}, K_{2}\right)$ означает, что $\mathcal{W} \in S^{\prime 0}\left(K_{1}, K_{2}, \mathbb{R}^{4}\right)$. Введем обозначение

$$
\mathbb{J}_{n-1}^{c}=\mathbb{V}_{c, R}^{n-1} \cup \mathbb{V}_{\mathrm{c}, L}^{n-1},
$$

где $\mathbb{V}_{\mathrm{c}, R}=\left\{\left(\xi^{0}, \xi^{1}\right):\left(\xi^{0}\right)^{2}-\left(\xi^{1}\right)^{2}<0, \xi^{1}>0\right\}, \mathbb{V}_{\mathrm{c}, L}=-\mathbb{V}_{\mathrm{c}, R}$. Согласно [1] двухкомпонентный открытый конус (15) состоит из вещественных точек аналитичности вайтмановских функций локальной теории поля в двумерном пространстве-времени.

Теорема 7. Пусть $\phi(x)$ - скалярное поле, определенное как операторная обобщенная функиия на пространстве $S^{0}\left(\mathbb{R}^{4}\right)$ и удовлетворяющее условиям (12), (14). Тогда клин $\complement \coprod_{n-1}^{c} \times \mathbb{R}^{2(n-1)}$ при любом $n \geqslant 2$ является строго несущим для разности

$$
W\left(\xi_{1}, \ldots, \xi_{n-1}\right)-W\left(-\xi_{1}, \ldots,-\xi_{n-1}\right)
$$

В частности, клин (13) является строго несущим для вакуумного среднего коммymamopa

$$
\left\langle\Psi_{0},\left[\phi(x), \phi\left(x^{\prime}\right)\right]_{-} \Psi_{0}\right\rangle .
$$

ДокАЗАТЕЛЬСтво. Функции Вайтмана инвариантны относительно одновременной инверсии координат $\xi^{2}$ и $\xi^{3}$, реализуемой поворотом на $180^{0}$ из группы $S O(2)$. Поэтому достаточно доказать, что указанный клин является строго несущим для разности

$$
W(\xi)-W\left(I_{\mathrm{c}} \xi\right)
$$

где $I_{\mathrm{c}}$ - инверсия коммутативных координат $\xi^{0}, \xi^{1}$, и для краткости использовано обозначение $\xi=\left(\xi_{1}, \ldots, \xi_{n-1}\right)$. Согласно теореме 4 это равносильно тому, что конус $\complement \rrbracket_{n-1}^{c}$ является несущим для всех функционалов, получаемых из (18) усреднением с пробными функциями, зависящими лишь от $\xi^{2}, \xi^{3}$. Пусть $W_{g}$ - результат усреднения $W$ с такой функцией $g \in S^{0}\left(\mathbb{R}^{2(n-1)}\right)$. Из условий теоремы следует, что 
функционал $W_{g} \in S^{\prime 0}\left(\mathbb{R}^{2(n-1)}\right)$ инвариантен относительно группы $S O_{0}(1,1)$ собственных ортохронных преобразований Лоренца в размерности $1+1$, и носитель его преобразования Фурье содержится в остром конусе $\overline{\mathbb{V}}_{c+}^{(n-1)}$. Если считать $W$ распределением умеренного роста, как принято в [9], то из этого свойства носителя следует, что $W_{g}$ является граничным значением функции $\mathbf{W}_{g}(\zeta)$, аналитической в трубе $\mathbb{T}_{\mathrm{c}-}^{n-1}=\left\{\zeta=\xi_{\mathrm{c}}+i \eta: \eta \in \mathbb{V}_{\mathrm{c}-}^{n-1}\right\}$. По теореме Баргмана-Холла-Вайтмана [1]-[3] функция $\mathbf{W}_{g}$ допускает аналитическое продолжение в расширенную трубу, которое инвариантно относительно собственной комплексной группы Лоренца. В двумерном случае эта группа совпадает с группой $\mathbb{C}^{*}=\mathbb{C} \backslash\{0\}$ комплексных чисел, и в координатах светового конуса $\zeta^{ \pm}=\left(\zeta^{0} \pm \zeta^{1}\right) / \sqrt{2}$ ее действие имеет вид

$$
\zeta^{ \pm} \rightarrow z^{ \pm 1} \zeta^{ \pm}, \quad z \in \mathbb{C}^{*}
$$

В частности, данная группа содержит инверсию $I_{c}$, хотя это преобразование не принадлежит компоненте единицы $S O(1,1)$. Очевидно, конус $(15)$ содержится в расширенной трубе ${ }^{2)}$, поскольку $\mathbb{V}_{\mathrm{c}, R}^{n-1}=\left\{\xi_{\mathrm{c}}: \xi^{+}>0, \xi^{-}<0\right\}$, и преобразование (19) с $z=-i$ переводит $\mathbb{V}_{\mathrm{c}, R}^{n-1}$ в $i \mathbb{V}_{\mathrm{c}-}^{n-1}$. Для $\mathbb{V}_{\mathrm{c}, L}^{n-1}$ ту же роль играет $z=i$. Поскольку выбор $g$ произволен, отсюда немедленно следует, что в некоммутативной теории умеренного роста [9] разность (18) равна нулю всюду в клине $\mathbb{J}_{n-1}^{\mathrm{c}} \times \mathbb{R}^{2(n-1)}$.

Ультрафиолетовое поведение $W_{g} \in S^{\prime 0}$ можно регуляризовать умножением его преобразования Фурье на $S O(1,1)$-инвариантную функцию $\omega\left(P_{\mathrm{c}}^{2} / \Lambda\right)$, где $\omega \in C_{0}^{\infty}(\mathbb{R})$ и $P_{\mathrm{c}}=\sum_{j} p_{\mathrm{c}, j}$. Теорема 8 в [19] показывает, что это дает распределение умеренного роста. Для краткости обозначим $g$-усредненную разность (18) через $G$, а результат ее регуляризации - через $G_{\Lambda}$. В силу сказанного выше $\operatorname{supp} G_{\Lambda}$ содержится в дополнении к конусу Йоста $\mathbb{J}_{n-1}^{c}$. Следовательно, это распределение при любом $\Lambda$ имеет непрерывное продолжение $\widehat{G}_{\Lambda}$ на пространство $S^{0}(U)$, где $U \ni \complement J_{n-1}^{c}$, и даже на $S^{0}(\mathbb{U})$, где $\mathbb{U}$ - внутренность замкнутого конуса $\complement \mathbb{J}_{n-1}^{c}$. Такое продолжение можно задать формулой $\left(\widehat{G}_{\Lambda}, f\right)=\left(G_{\Lambda}, \chi f\right)$, где $\chi$ - бесконечно дифференцируемая функция, равная единице в $\epsilon$-окрестности $\mathbb{U}$ и обращающаяся в нуль вне $2 \epsilon$-окрестности. Ясно, что умножение на $\chi$ непрерывно отображает $S^{0}(\mathbb{U})$ в пространство Шварца $S\left(\mathbb{R}^{2(n-1)}\right)$. Далее применима аргументация, использованная при выводе СРТтеоремы для нелокальной КТП в [13]. Именно, выберем $\omega$ так, чтобы $\omega(t)=1$ при $|t| \leqslant 1$, и определим функционал $\widehat{G}$ на $S^{0}(\mathbb{U})$, полагая

$$
(\widehat{G}, f)=\left(\widehat{G}_{\Lambda}, f\right), \quad \text { где } \quad \Lambda=4 \sqrt{2 n^{3}} e b, \quad \text { если } f \in S^{0, b}(\mathbb{U}) .
$$

Теорема 1 обеспечивает корректность определения функционала $\widehat{G}$. Согласно ее подробной формулировке, данной в [13], пространство $S^{0, b^{\prime}}\left(\mathbb{R}^{d}\right)$ плотно в $S^{0, b}(U)$ в топологии $S^{0, b^{\prime}}(U)$ для любого открытого конуса $U \subset \mathbb{R}^{d}$ и для любой пары положительных чисел $b, b^{\prime}$ таких, что $b^{\prime}>2 d e b$. Поэтому существует последовательность функций $f_{\nu} \in S^{0,4 n e b}\left(\mathbb{R}^{2(n-1)}\right)$ такая, что $f_{\nu} \rightarrow f$ в $S^{0,4 n e b}(\mathbb{U})$. Согласно теореме Пэли-Винера-Шварца (теореме 7.3.1 в [18]) носители преобразований Фурье функций $f_{\nu}$ содержатся в гиперкубе $\max _{j}\left(\left|p_{j}^{0}\right|,\left|p_{j}^{1}\right|\right) \leqslant 4 n e b$, где $\left|P_{\mathrm{c}}^{2}\right| \leqslant 32 n^{3} e^{2} b^{2}$

\footnotetext{
2) Отметим, что в [1] описание вещественных точек аналитичности в любой размерности как раз и сводится к этому простому двумерному случаю.
} 
и $\omega\left(P_{\mathrm{c}}^{2} / \Lambda^{2}\right) \equiv 1$ при указанном выборе $\Lambda$. Поэтому если $b_{1}>b$, то $\left(\widehat{G}_{\Lambda}, f_{\nu}\right)=$ $\left(\widehat{G}_{\Lambda_{1}}, f_{\nu}\right)=\left(G, f_{\nu}\right)$ и, значит, $\left(\widehat{G}_{\Lambda}, f\right)=\left(\widehat{G}_{\Lambda_{1}}, f\right)$. Это же рассуждение показывает, что $\widehat{G}$ является продолжением $G$, непрерывным в топологии $S^{0}(\mathbb{U})$. Тем самым доказательство закончено.

Теорема 7 показывает, что при использовании слабой формы спектрального условия (12) и пробных функций класса $S^{0}$ подходящей модификацией аксиомы микропричинности служит требование того, чтобы клин (13) являлся строго несущим для матричных элементов $\left\langle\Phi,\left[\phi_{\iota}(x), \phi_{\iota^{\prime}}\left(x^{\prime}\right)\right]_{\mp} \Psi\right\rangle$ коммутаторов или антикоммутаторов полей теории при любых $\Phi$ и $\Psi$ из общей плотной инвариантной области $D$ определения полей в пространстве состояний.

Теперь покажем, что установленная в [13] теорема единственности для распределений с носителем в остром конусе допускает прямое обобщение на случай клинообразного носителя.

Теорема 8. Пусть $u \in \mathcal{D}^{\prime}\left(\mathbb{R}^{d_{1}} \times \mathbb{R}^{d_{2}}\right)$ - нетривиальное распределение с носителем в клине $V \times \mathbb{R}^{d_{2}}$, где $V$ - острый конус. Тогда любой строго несущий конус его преобразования Фуръе $\tilde{u} \in S^{\prime 0}\left(\mathbb{R}^{d_{1}} \times \mathbb{R}^{d_{2}}\right)$ имеет вид $\mathbb{R}^{d_{1}} \times K_{2}$, где $K_{2}$ - замкнутый конус в $\mathbb{R}^{d_{1}}$.

ДокАЗАТЕЛЬСтво. Предположим, что это не так: $\tilde{u} \in S^{\prime 0}\left(K_{1}, K_{2}\right)$, где $K_{1} \neq$ $\mathbb{R}^{d_{1}}$. Тогда $K_{1}$ является несущим конусом любого функционала $\tilde{u}_{g}, g \in S^{0}\left(\mathbb{R}^{d_{2}}\right)$, определяемого формулой $\left(\tilde{u}_{g}, f\right)=(\tilde{u}, f \otimes g)$, что становится очевидным, если учесть формулу (2). Обратное преобразование Фурье функционала $\tilde{u}_{g}$ есть распределение на $\mathbb{R}^{d_{1}}$ с носителем в $V$, и согласно теореме 4 в [13] это распределение тривиально. Поскольку функция $g$ произвольна, а $\mathcal{D}\left(\mathbb{R}^{d_{1}}\right) \otimes \mathcal{D}\left(\mathbb{R}^{d_{2}}\right)$ плотно в $\mathcal{D}\left(\mathbb{R}^{d_{1}} \times \mathbb{R}^{d_{2}}\right)$, мы заключаем, что $u \equiv 0$, и приходим к противоречию.

Теорема 8 позволяет распространить на обсуждаемый вариант некоммутативной теории поля часть результатов работ [5], [13], касающихся связи спина со статистикой и СРТ-симметрии. Пусть

$$
W_{\phi \phi^{*}}\left(x-x^{\prime}\right)=\left\langle\Psi_{0}, \phi(x), \phi^{*}\left(x^{\prime}\right) \Psi_{0}\right\rangle, \quad W_{\phi^{*} \phi}\left(x-x^{\prime}\right)=\left\langle\Psi_{0}, \phi^{*}(x), \phi\left(x^{\prime}\right) \Psi_{0}\right\rangle .
$$

В качестве простейшего примера покажем, что клин (13) не может быть строго несущим для антикоммутатора $\left[\phi(x), \phi^{*}\left(x^{\prime}\right)\right]_{+}$, т.е. аномальное коммутационное соотношение для скалярного поля запрещено. Действительно, в противном случае клин $\overline{\mathbb{V}}_{\mathrm{c}} \times \mathbb{R}^{2}$ является в силу теоремы 7 строго несущим для функционала $W_{\phi \phi^{*}}+W_{\phi^{*} \phi}$. Из (12) следует, что в импульсном представлении носитель этой суммы лежит в клине $\overline{\mathbb{V}}_{\mathrm{c}}+\times \mathbb{R}^{2}$. Значит, сумма равна нулю по теореме 8 . Усредняя с пробной функцией вида $\bar{f}(x) f\left(x^{\prime}\right)$, получаем $\left\|\phi^{*}(f) \Psi_{0}\right\|^{2}+\left\|\phi(f) \Psi_{0}\right\|^{2}=0$ и, следовательно,

$$
\phi(f) \Psi_{0}=\phi^{*}(f) \Psi_{0}=0 \quad \text { для всех } f \in S^{0}\left(\mathbb{R}^{4}\right) .
$$

Как и в обычной локальной теории [2], [3], отсюда следует тождественное равенство поля $\phi$ нулю, что устанавливается с помощью аргументации, аналогичной той, которая применяется при выводе теоремы 13 в [5], и снова использующей полученное обобщение теоремы единственности. 
Как известно, в теории скалярного поля наличие СРТ-симметрии равносильно соблюдению соотношений

$$
W\left(\xi_{1}, \ldots, \xi_{n-1}\right)=\breve{W}\left(\xi_{n-1}, \ldots, \xi_{1}\right)
$$

где $\breve{W}$ определяется тем же набором операторов, что и $W$, но взятым в обратном порядке. Запишем функционал

$$
W\left(\xi_{1}, \ldots, \xi_{n-1}\right)-\breve{W}\left(\xi_{n-1}, \ldots, \xi_{1}\right)
$$

в виде суммы

$$
\left[W\left(\xi_{1}, \ldots, \xi_{n-1}\right)-\breve{W}\left(-\xi_{n-1}, \ldots,-\xi_{1}\right)\right]+\left[\breve{W}\left(-\xi_{n-1}, \ldots,-\xi_{1}\right)-\breve{W}\left(\xi_{n-1}, \ldots, \xi_{1}\right)\right] .
$$

Согласно теореме 7 клин $\complement \mathbb{J}_{n-1}^{c} \times \mathbb{R}^{2(n-1)}$ является строго несущим для функционала во вторых квадратных скобках в (23). Если модифицированное условие причинности выполнено с нормальным коммутационным соотношением, то этот клин является строго несущим и для функционала в первых квадратных скобках в (23). Действительно, этот функционал выражает разность двух вакуумных средних, получаемых одно из другого перестановкой полей. Эта разность представима как сумма членов вида $\left\langle\Psi_{0}, \ldots\left[\phi^{(*)}\left(x_{i}\right), \phi^{(*)}\left(x_{j}\right)\right]_{-} \ldots \Psi_{0}\right\rangle$, где точки символизируют полевые операторы. Для такого члена клин $\overline{\mathbb{V}}_{\mathrm{c}} \times \mathbb{R}^{4 n-2}=\left\{x:\left(x_{i}-x_{j}\right)_{\mathrm{c}}^{2} \geqslant 0\right\}$ является строго несущим, а его образ $\left\{\xi:\left(\xi_{i}+\cdots+\xi_{j-1}\right)_{\mathrm{c}}^{2} \geqslant 0\right\}$ в пространстве относительных координат содержится в $\complement \mathbb{J}_{n-1}^{c} \times \mathbb{R}^{2(n-1)}$. Из (12) следует, что в импульсном представлении носитель функционала (22) лежит в клине $\overline{\mathbb{V}}_{\mathrm{c}+}^{n-1} \times \mathbb{R}^{2(n-1)}$. Снова применяя теорему 8 , заключаем, что некоммутативная теория скалярного поля с функциональной областью определения $S^{0}\left(\mathbb{R}^{4}\right)$, удовлетворяющая спектральному условию (12) и сформулированному выше модифицированному условию причинности, обладает СРТ-симметрией. Более того, существование оператора СРТ-симметрии равносильно тому, что клинья $\complement J_{n-1}^{c} \times \mathbb{R}^{2(n-1)}, n=2,3, \ldots$, являются строго несущими для функционалов в первых квадратных скобках в (23).

\section{6. ЗАКЛЮЧЕНИЕ}

Установленная эквивалентность асимптотической коммутативности и свойств регулярности запаздывающих функций Грина в $p$-пространстве еще раз показывает, что формулировка нелокальной КТП, использующая высокосингулярные обобщенные функции, достаточно содержательна с физической точки зрения, поскольку допускает последовательную интерпретацию в терминах рассеяния частиц, при этом имеют место нормальная связь спина со статистикой и СРТ-инвариантность. Следует отметить, что отличие строго несущих конусов от несущих конусов характерно не только для функционалов класса $S^{\prime 0}$. Оно присуще и обобщенным функциям, определенным на любом пространстве Гельфанда-Шилова $S^{\beta}$ или $S_{\alpha}^{\beta}$ с индексом $\beta \leqslant 1$. В частности, это относится к фурье-гиперфункциям, которые составляют пространство, сопряженное к $S_{1}^{1}$, и дают наиболее широкие рамки для построения 
локальной теории поля. Мы прокомментировали здесь лишь одну из попыток распространения аксиоматического подхода на некоммутативные теории поля. Представляет интерес рассмотреть возможность использования аналитических пробных функций и в других формулировках, в частности, при определении вайтмановских функций с заменой обычного произведения полей мояловским *-произведением и с реализацией симметрии относительно деформированной алгебры Пуанкаре, предлагавшейся в работе [20], но это выходит за рамки данной статьи.

Благодарности. Работа поддержана РФФИ (грант № 05-01-01049) и Программой поддержки ведущих научных школ (грант № НШ-1578.2003.2).

\section{Список литературы}

[1] R. Jost, The General Theory of Quantized Fields, AMS, Providence, RI, 1965.

[2] Р. Стритер, А. Вайтман, РCT, спин и статистика и все такое, Наука, М., 1966.

[3] Н. Н. Боголюбов, А. А. Логунов, А. И. Оксак, И. Т. Тодоров, Общие принципь квантовой теории поля, Наука, М., 1987.

[4] O. Steinmann, Commun. Math. Phys., 18 (1970), 179.

[5] М. А. Соловьев, ТМФ, 121 (1999), 139.

[6] М. А. Соловьев, ТMФ, 143 (2005), 195.

[7] B. Schroer, Ann. Phys., 319 (2005), 92.

[8] N. Seiberg, E. Witten, JHEP, 9909 (1999), 032.

[9] L. Álvarez-Gaumé, M. A. Vázquez-Mozo, Nucl. Phys. B, 668 (2003), 293.

[10] C.-S. Chu, K. Furuta, T. Inami, Int. J. Mod. Phys. A, 21 (2006), 67; hep-th/0502012.

[11] V. E. Hubeny, M. Rangamani, S. F. Ross, JHEP, 0507 (2005), 037.

[12] D. H. T. Franco, C. M. M. Polito, J. Math. Phys., 46 (2005), 083503.

[13] M. A. Soloviev, J. Math. Phys., 39 (1998), 2635.

[14] И. М. Гельфанд, Г. Е. Шилов, Обобщенные функиии, m. 2, Физматлит, М., 1958.

[15] A. G. Smirnov, J. Math. Phys., 44 (2003), 2058.

[16] M. A. Soloviev, J. Math. Phys., 45 (2004), 1944.

[17] M. A. Soloviev, Carrier cones of analytic functionals, math-ph/0507011.

[18] Л. Хермандер, Анализ линейных дифференциалъных операторов с частными производными, m. 1, Мир, М., 1986.

[19] М. А. Соловьев, ТMФ, 128 (2001), 492.

[20] M. Chaichian, P. P. Kulish, K. Nishijima, A. Tureanu, Phys. Lett. B, 604 (2004), 98. 